Article

\title{
Crystal Transition Behavior and Thermal Properties of Thermal-Energy-Storage Copolymer Materials with an $n$-Behenyl Side-Chain
}

\author{
Yuchen Mao ${ }^{1,2} \mathbb{D}$, Jin Gong ${ }^{1,3, * \mathbb{D}}$, Meifang $\mathrm{Zhu}^{2, *}$ and Hiroshi Ito ${ }^{1, * \mathbb{C}}$ \\ 1 Department of Polymer Science and Engineering, Graduate School of Organic Materials Science, Yamagata \\ University, 4-3-16 Jonan, Yonezawa, Yamagata 992-8510, Japan; maoyc91@gmail.com \\ 2 State Key Laboratory for Modification of Chemical Fibers and Polymer Materials, College of Materials \\ Science and Engineering, Donghua University, Shanghai 201620, China \\ 3 Department of Mechanical Systems Engineering, Graduate School of Science and Engineering, Yamagata \\ University, 4-3-16 Jonan, Yonezawa, Yamagata 992-8510, Japan \\ * Correspondence: jingong@yz.yamagata-u.ac.jp (J.G.); zmf@dhu.edu.cn (M.Z.); \\ ihiroshi@yz.yamagata-u.ac.jp (H.I.); \\ Tel.: +81-238-26-3135 (J.G.); +86-21-6779-2865 (M.Z.); +81-238-26-3081 (H.I.)
}

Received: 19 July 2019; Accepted: 14 September 2019; Published: 17 September 2019

\begin{abstract}
In this paper, we synthesized MC(BeA-co-MMA) copolymer microcapsules through suspension polymerization. The pendent $n$-behenyl group of BeA is highly crystalline, and it acts as the side-chain in the structure of BeA-co-MMA copolymer. The highly crystalline $n$-behenyl side-chain provides BeA-co-MMA copolymer thermal-energy-storage capacity. In order to investigate the correlation between the thermal properties and crystal structure of the BeA-co-MMA copolymer, the effects of monomer ratio, temperature changing and the changing rate, as well as synthesis method were discussed. The monomer ratio influenced crystal transition behavior and thermal properties greatly. The DSC results proved that when the monomer ratio of BeA and MMA was 3:1, MC(BeA-co-MMA) 3 showed the highest average phase change enthalpy $\Delta H\left(105.1 \mathrm{~J} \cdot \mathrm{g}^{-1}\right)$. It indicated that the $n$-behenyl side-chain formed a relatively perfect crystal region, which ensured a high energy storage capacity of the copolymer. All the DSC and SAXS results proved that the amount of BeA had a strong effect on the thermal-energy-storage capacity of the copolymer and the long spacing of crystals, but barely on the crystal lamella. It was found that MMA units worked like defects in the $n$-behenyl side-chain crystal structure of the BeA-co-MMA copolymer. Therefore, a lower fraction of MMA, that is, a higher fraction of BeA, contributed to a higher crystallinity of the BeA-co-MMA copolymer, providing a better energy storage capacity and thermoregulation property. ST(BeA-co-MMA) copolymer sheets with the same ingredients as microcapsules were also prepared through light-induced polymerization aiming at clarifying the effect of the synthesis method. The results proved that the synthesis method mainly influenced the copolymer chemical component, but lightly on the crystal packing of the $n$-behenyl side-chain.
\end{abstract}

Keywords: crystal transition behavior; thermal property; thermal energy storage; copolymer; suspension polymerization; light-induced polymerization

\section{Introduction}

A latent heat storage system is a highly efficient and environmentally friendly means to use residual heat, recovering waste heat and to store/release thermal energy for saving energy. Phase change materials (PCMs) are considered to be among the most reliable latent heat storage and thermoregulation materials, which work by absorbing or releasing the enthalpy of phase changes in certain temperature 
ranges. PCMs have been successfully utilized in aerospace and aviation, solar engineering, building air conditioning, insulating clothing and textiles, preservation and environmental temperature-control since their developments in the 1980s [1-6].

Polymeric PCMs transform from one semi-crystalline state into another semi-crystalline state, or an amorphous state, with the advantages of small volume change, high energy-storage density, great design flexibility and form-stable at a temperature above the melting point [7-9]. Polymeric PCMs also benefit from less phase segregation and good physical-chemical stability. These features give them perfect thermal durability and cycling stability against thermal degradation required for a long-term service [10-13]. Comb-like polymers, with a crystalline long $n$-alkane side-chain attached to a main-chain, are known to pack into a layered structure with alternating side-chain crystal regions at room temperature [14-17]. In our group, much of the work revolves about crystalline copolymer microcapsules and gels having a chemical structure with an $n$-alkane crystalline side-chain. The behenyl acrylate (BeA) consisted of an $n$-behenyl chain with 22 methylene units and a vinyl group was used as the phase change monomer to synthesize $\mathrm{P}(\mathrm{BeA}-c o-\mathrm{MMA})$ copolymer microcapsules with methyl methacrylate (MMA) through suspension polymerization [18]. Furthermore, we have developed crystalline gels with high toughness and high flexibility, as well as other functions such as shape memory, humidity regulation, thermal expansion and thermal energy storage [19-24]. We have confirmed that these copolymers can form crystal regions at room temperature to provide thermoregulation properties, and their application potential as form-stable solid-solid PCMs are greatly expected.

For the crystalline polymers, crystallization controls the assembly of ordered crystalline structures from molecular chains, which determines the basic physical properties of crystalline polymer materials [25]. Therefore, in this paper, we aim to investigate the phase transition behavior between the crystal phase and the isotropic phase of the thermal-energy-storage copolymer materials with a crystalline $n$-behenyl side-chain. A series of copolymer microcapsules were synthesized from BeA and MMA, shorted as MC(BeA-co-MMA). Polymethyl methacrylate (PMMA) is a tough and rigid plastic having pendent methyl groups $\left(-\mathrm{CH}_{3}\right)$, which prevent the polymer chains from rotating freely around a carbon-carbon bond and packing closely in a crystal pattern. The introduction of MMA would change the chain flexibility and segment motion. Moreover, copolymer sheets with the same ingredients as copolymer microcapsules, shorted as ST(BeA-co-MMA), were synthesized as comparison samples through light-induced polymerization [20]. The synthesis schemes of the MC(BeA-co-MMA) copolymer are shown in Figure 1a, and the microcapsules and sheets prepared through suspension and light-induced polymerization, respectively, are presented in Figure $1 \mathrm{~b}$. The effects of monomer ratio and synthesis method on the crystal transition behavior and thermal properties were studied. 


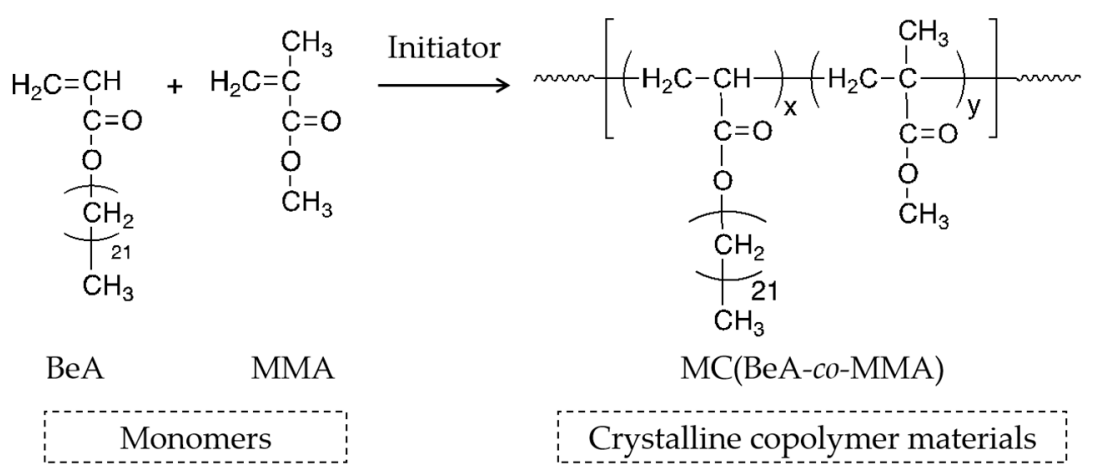

(a)

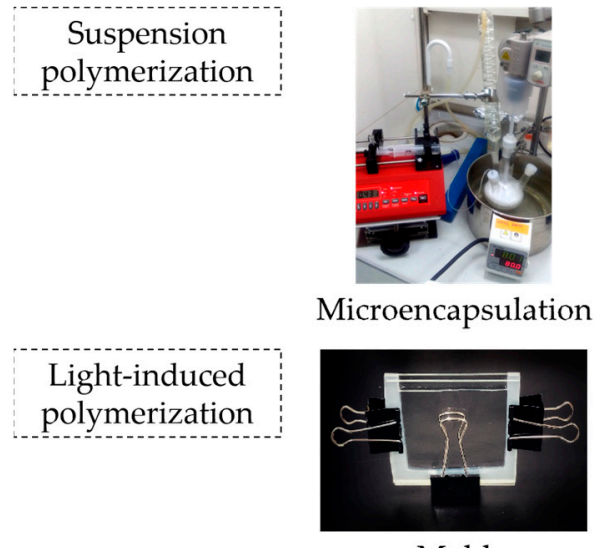

Mold

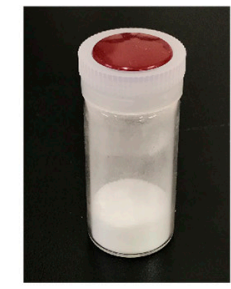

MC(BeA-co-MMA)3

UV irridiation

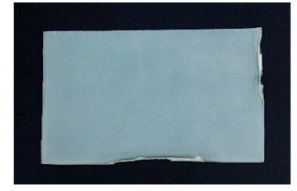

ST(BeA-co-MMA)3

(b)

Figure 1. Synthesis schemes of thermoregulation MC(BeA-co-MMA) copolymer (a), and the microcapsules and sheets prepared through suspension and light-induced polymerization, respectively (b). The phase transition between the crystal phase and the isotropic phase promises the energy storage capacity.

\section{Materials and Experiments}

\subsection{Materials}

Monomer BeA was provided by Shin-Nakamura Chemical Co., Ltd., Wakayama, Japan. Monomer MMA, thermal initiator 2,2' -azobis (2-methylpropionitrile) (AIBN), photo initiator benzophenone (BP) and methanol were purchased from Wako Pure Chemical Industries, Ltd., Tokyo, Japan. Polyvinyl alcohol (PVA) provided by Kuraray Co., Ltd., Okayama, Japan, was used as an emulsifier. All the above reagents were used without further purification.

\subsection{Preparation of Copolymer Microcapsules}

Copolymer microcapsules were synthesized by free radical suspension polymerization in an emulsion system with an oil-soluble initiator. Random copolymerization was carried out with varying monomer mass ratios between BeA and MMA in a similar manner as we reported before [18]. Crystalline copolymer microcapsules MC(BeA-co-MMA)1, MC(BeA-co-MMA)3 and MC(BeA-co-MMA)5 with monomer mass ratios of two associated monomers from 1:1, 3:1 to 5:1 were synthesized (listed in Table 1). Heat-initiated poly(methyl methacrylate) and poly(behenyl acrylate), named MC(MMA) and $\mathrm{MC}(\mathrm{BeA})$, respectively, were prepared and evaluated too. 
Table 1. Composition of all the thermal-energy-storage copolymer microcapsules and sheets with a crystalline $n$-behenyl side-chain synthesized in this work by varying monomer mass ratios.

\begin{tabular}{ccccc}
\hline \multicolumn{2}{c}{ Sample } & Synthesis Method & Monomers & Monomer Ratio \\
\hline \multirow{4}{*}{ Microcapsule } & & MMA & - \\
& MC(MMA) & Heat-initiated & BeA & - \\
& MC(BeA-co-MMA)1 & suspension & BeA:MMA & $1: 1$ \\
& MC(BeA-co-MMA)3 & polymerization & BeA:MMA & $3: 1$ \\
MC(BeA-co-MMA)5 & & BeA:MMA & $5: 1$ \\
STheet & ST(BeA) & & MMA & - \\
& ST(BeA-co-MMA)1 & Light-induced & BeA & - \\
& ST(BeA-co-MMA)3 & polymerization & BeA:MMA & $1: 1$ \\
& ST(BeA-co-MMA)5 & & BeA:MMA & $3: 1$ \\
& & & BeA:MMA & $5: 1$ \\
\hline
\end{tabular}

\subsection{Preparation of Copolymer Sheets}

For a comparison to microcapsules, we synthesized copolymer sheets with an $n$-behenyl side-chain using the same ingredients with the monomer mass ratios of BeA to MMA at 1:1, 3:1 and 5:1, marked ST(BeA-co-MMA)1, ST(BeA-co-MMA)3 and ST(BeA-co-MMA)5, respectively (listed in Table 1). The reactive uncured liquid resin consisted of BeA, MMA and photo initiator BP. BP was dissolved in MMA firstly, and melted BeA was added to make a homogeneous resin. Then, the liquid resin was injected into a mold with $1 \mathrm{~mm}$ thickness (Figure $1 \mathrm{~b}$ ), and cured under UV irradiation for $48 \mathrm{~h}$ at $30{ }^{\circ} \mathrm{C}$. The UV lamp type, power and wavelength are the key factors for the UV irradiation process. The UV lamps with a peak wavelength of $365 \mathrm{~nm}$ were purchased from Toshiba Lighting \& Technology Corporation (Yokosuka, Japan). The UV intensity varies depending on the irradiation distance. The intensity of UV reaching samples ranged from 200 to $300 \mu \mathrm{W} \cdot \mathrm{cm}^{-2}$. Light-induced poly(methyl methacrylate) and poly(behenyl acrylate) sheets, named ST(MMA) and ST(BeA), were prepared in a similar manner.

All the thermal-energy-storage copolymer microcapsules and sheets with a crystalline $n$-behenyl side-chain synthesized in this work are listed in Table 1.

\subsection{Characterization of Copolymer Microcapsules and Sheets}

\subsubsection{Differential Scanning Calorimetry (DSC)}

The thermal properties and phase transition behavior of copolymers were investigated using a differential scanning calorimeter (Q-2000, TA instruments Japan Inc., Tokyo, Japan) operating under a nitrogen flow. Samples of about $5 \mathrm{mg}$ were heated to $80^{\circ} \mathrm{C}$ at a heating rate of $5{ }^{\circ} \mathrm{C} \cdot \mathrm{min}^{-1}$, and held for $1 \mathrm{~min}$ to eliminate the thermal prehistory. Then, samples were cooled down to $0^{\circ} \mathrm{C}$ with various cooling rates for measuring their crystal behavior. After holding for $1 \mathrm{~min}$, samples were reheated up to $80^{\circ} \mathrm{C}$ at the same cooling rate. Premium hermetic pans (TA Instruments Tzero \#901683.901, TA instruments Japan Inc., Tokyo, Japan) were used for the measurements.

\subsubsection{Small-Angle X-ray Scattering (SAXS)}

The crystal transition behavior and crystal structures of copolymer microcapsules were analyzed using temperature-variable, small-angle X-ray scattering (Nano-Viewer, Rigaku Corporation, Akishima, Japan) with a nickel-filtered $\mathrm{Cu}-K \alpha$ radiation (wavelength $\lambda=1.5405 \AA$ ), and a high-speed two-dimensional imaging plate detector (Pilatus3 R 100K, Rigaku Corporation, Akishima, Japan). In order to detect and analyze the transition behavior of the side-chain and copolymer skeleton in the crystallization process, the camera distance was set to $0.1 \mathrm{~m}$. The effective detection range can expand to $2 \theta=30^{\circ}$. All measurements were carried out within the exposure time of $5 \mathrm{~min}$ and at a heating/cooling rate of $0.5^{\circ} \mathrm{C} \cdot \mathrm{min}^{-1}$. The heating-cooling cycles of microcapsules were taken with the temperature ranging from 35 to $70{ }^{\circ} \mathrm{C}$ on a hot stage (FP82HT hot stage connected to FP90 central 
processor, Mettler Toledo, Tokyo, Japan). Diffraction diagrams recorded on the flat imaging plate detector by the beam-transmission technique were converted to the one-dimensional profile against diffraction angle $2 \theta$ through Igor Pro 7 (WaveMetrics Inc., Lake Oswege, OR, USA) with a special add-in progress. The interplanar crystal spacing $d$ was calculated by the following formula:

$$
d=\frac{\lambda}{2 \sin \theta}
$$

\subsubsection{Wide-Angle X-ray Scattering (WAXS)}

To determine the crystal structure and crystallinity of copolymer materials, wide-angle X-ray scattering was performed on an X-ray diffractometer (Ultima IV, Rigaku Corporation, Akishima, Japan) with nickel-filtered $\mathrm{Cu}-K \alpha$ radiation (wave length $\lambda=1.5405 \AA$ ) and a high-speed one-dimensional detector (D/tex Ultra, Rigaku Corporation, Akishima, Japan) at a scanning rate of $5^{\circ} \cdot \mathrm{min}^{-1}$. The crystallinity $\left(W_{\mathrm{c}}, \%\right)$ was evaluated according to the following formula [26,27]:

$$
W_{\mathrm{c}}=\frac{I_{\mathrm{c}}}{I_{\mathrm{c}}+I_{\mathrm{a}}} \times 100 \%
$$

where $I_{\mathrm{c}}$ and $I_{\mathrm{a}}$ are the scattering intensities of the crystalline region and amorphous region, respectively.

\subsubsection{Fourier Transform Infrared Spectrometer (FTIR)}

The chemical composition and structure of copolymer materials were evaluated using a Fourier transform infrared spectrometer (FT/IR-460 Plus, JASCO International Co., Ltd., Tokyo, Japan). The spectra were recorded with 32 scans in transmittance mode with a resolution of $0.5 \mathrm{~cm}^{-1}$ within the range of 1000 to $3200 \mathrm{~cm}^{-1}$.

\section{Results and Discussion}

\subsection{Effect of Monomer Ratio}

The crystal transition behavior of microcapsules determines the thermal properties and energy storage capacities. Figure 2 shows the DSC curves of MC(BeA-co-MMA) microcapsules with an $n$-behenyl side-chain in the cooling process, measured at a cooling rate of $5{ }^{\circ} \mathrm{C} \cdot \mathrm{min}^{-1}$. The phase transition temperatures and phase change enthalpy are listed in Table 2. $\mathrm{MC}(\mathrm{BeA})$ had the strongest sharp exothermic peak with the highest phase change enthalpy. It indicated a quick transition from the isotropic phase to the crystal phase, and the great potential to be used as a thermal-energy-storage material. MC(MMA) was amorphous so it did not show any exothermic peaks in the cooling process. Compared to MC(MMA), MC(BeA-co-MMA) copolymer microcapsules showed an exothermic peak thanks to the introduction of the phase change component BeA. The peak was corresponding to crystallization of the $n$-behenyl side-chains. In the comb-like copolymer, eight or nine methylene units of the side-chain in the vicinity of the main-chain are amorphous. It is confirmed that the polymers of octadecyl and hexadecyl methacrylates crystallize with the terminal parts of 9-10 units and $7-8$ units. $[28,29]$. In the cooling process, the segment motion of the crystalline $n$-behenyl side-chain was restricted by the rigid copolymer skeleton. Crystalline side-chains did not have enough mobility to adjust the steric conformation to allow all methylene units to arrange in crystals. Thus, only the terminal parts of the $n$-behenyl side-chain could form crystal regions, which led to a weaker and broader exothermic peak for MC(BeA-co-MMA) copolymer microcapsules. 


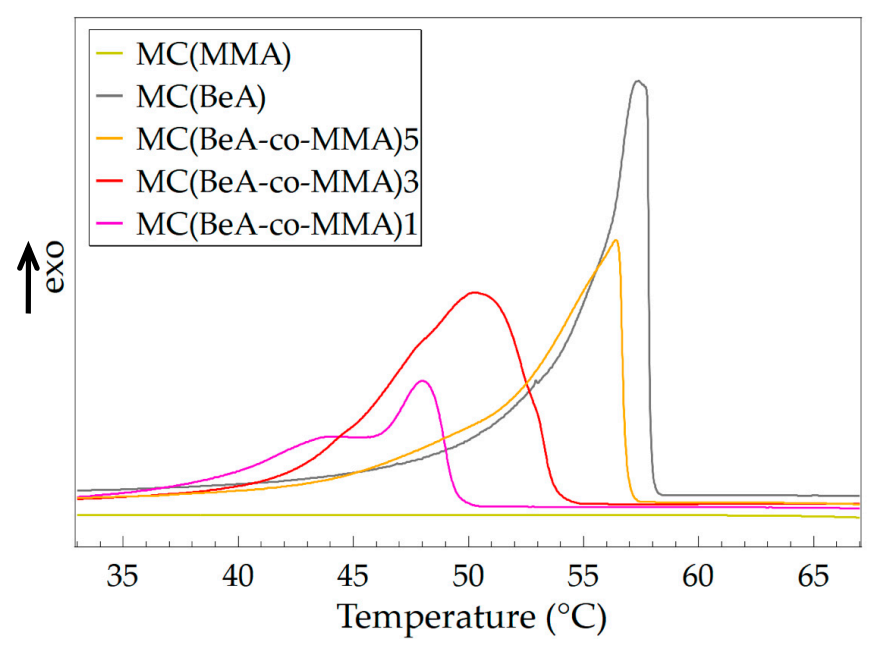

Figure 2. DSC curves of $\mathrm{MC}(\mathrm{BeA}-\mathrm{co}-\mathrm{MMA})$ copolymer microcapsules and $\mathrm{MC}(\mathrm{BeA})$ in the cooling process measured at a cooling rate of $5^{\circ} \mathrm{C} \cdot \mathrm{min}^{-1}$.

Table 2. Thermal properties of $\mathrm{MC}(\mathrm{BeA})$ and $\mathrm{MC}(\mathrm{BeA}-\mathrm{co}-\mathrm{MMA})$ copolymer microcapsules with different monomer ratios, measured at a temperature changing rate of $5{ }^{\circ} \mathrm{C} \cdot \mathrm{min}^{-1}$.

\begin{tabular}{cccccc}
\hline \multirow{2}{*}{ Sample } & \multicolumn{2}{c}{ Crystallization } & \multicolumn{2}{c}{ Melting } & \multirow{2}{c}{$\begin{array}{c}\text { Degree of } \\
\text { Supercooling }\left({ }^{\circ} \mathbf{C}\right)\end{array}$} \\
\cline { 2 - 4 } & $\boldsymbol{T}_{\mathbf{c}}\left({ }^{\circ} \mathbf{C}\right)$ & $\boldsymbol{\Delta H}_{\mathbf{c}}\left(\mathbf{J} \cdot \mathbf{g}^{-\mathbf{1}}\right)$ & $\boldsymbol{T}_{\mathbf{m}}\left({ }^{\circ} \mathbf{C}\right)$ & $\boldsymbol{\Delta H}_{\mathbf{m}}\left(\mathbf{J} \cdot \mathbf{g}^{-\mathbf{1}}\right)$ & - \\
MC(MMA) & - & - & - & - & 4.4 \\
MC(BeA) & 57.4 & 115.6 & 61.8 & 114.3 & 5.4 \\
MC(BeA-co-MMA)5 & 56.4 & 98.2 & 61.8 & 97.0 & 6.9 \\
MC(BeA-co-MMA)3 & 50.4 & 105.2 & 57.3 & 104.9 & 6.1 \\
MC(BeA-co-MMA)1 & 48.0 & 56.1 & 54.1 & 56.5 & - \\
\hline
\end{tabular}

$T_{\mathrm{c}}$ : crystallization temperature; $\Delta H_{\mathrm{c}}$ : enthalpy of crystallization process; $T_{\mathrm{m}}$ : melting temperature; $\Delta H_{\mathrm{m}}$ : enthalpy of melting process; Degree of supercooling: temperature difference between $T_{\mathrm{m}}$ and $T_{\mathrm{c}}\left(\Delta T=T_{\mathrm{m}}-T_{\mathrm{c}}\right)$. Enthalpy values during crystallization and melting can be considered identical within instrumental errors.

When the monomer ratio of BeA and MMA was 5:1, the crystallization temperature was only about $1{ }^{\circ} \mathrm{C}$ lower than that of $\mathrm{MC}(\mathrm{BeA})$. Additionally, the average phase change enthalpy $\Delta H$ of $\Delta H_{\mathrm{C}}$ and $\Delta H_{\mathrm{m}}$ was $97.6 \mathrm{~J}^{-1}{ }^{-1}$ for MC(BeA-co-MMA) 5 and $105.1 \mathrm{~J} \cdot \mathrm{g}^{-1}$ for MC(BeA-co-MMA)3, respectively. This indicated that the $n$-behenyl side-chain of MC(BeA-co-MMA) microcapsules could form a relatively perfect crystal region ensuring a high energy storage capacity. When the monomer ratio of BeA to MMA was 1:1, there existed two exothermic peaks. Many investigations on the crystallization behavior of $n$-alkane indicate that there are metastable rotator phases before the isotropic liquid completely converted into a crystalline solid [30,31]. Rotator phases pass through one or more than one rotator phases between the isotropic and crystal phase, due to the gradual breakdown of orientation order. As the amount of MMA was increasing, a stronger restriction was caused by the copolymer skeleton, which contributed to the peak appearing at $43^{\circ} \mathrm{C}$.

SAXS were carried out at room temperature for MC(BeA-co-MMA) microcapsules and MC(BeA), and the SAXS patterns are shown in Figure 3. The locations of diffraction peaks along with their corresponding interplanar crystal spacing $d$ are listed in Table 3. MC(BeA-co-MMA) microcapsules could provide an energy storage capacity because of the crystal transition of the $n$-behenyl side-chain. Three peaks were observed at diffraction angles $2 \theta=2.72^{\circ}, 4.58^{\circ}$ and $21.95^{\circ}$ on the SAXS pattern for $\mathrm{MC}(\mathrm{BeA})$. The peak I at the small angle region was assigned to the layered structure of the alternating crystalline side-chain regions and amorphous regions. The interplanar crystal spacing $d$ of Peak I and Peak II corresponded to the distance between copolymer chains separated by the side-chains. Researchers indicate that the long $n$-alkane chain compounds tend to crystallize into a lamellar structure, as the monolayer assemblies piled on one another, to arrange the functional groups regularly in each 
lamellar plane [28,32]. The peak I at $2.72^{\circ}$ indicated the long spacing of $32.45 \AA$, which was nearly equal to the length of the fully extended BeA with a $n$-behenyl chain. It suggested that the side-chains of the comb-like copolymer seemed to be aligned perpendicularly to the basal plane. The intense diffraction Peak III at $2 \theta=21.95^{\circ}$ corresponded to the spacing of $4.05 \AA$, which was considered the characteristic peak of the hexagonal packing of $n$-behenyl side-chains [14,32-34].

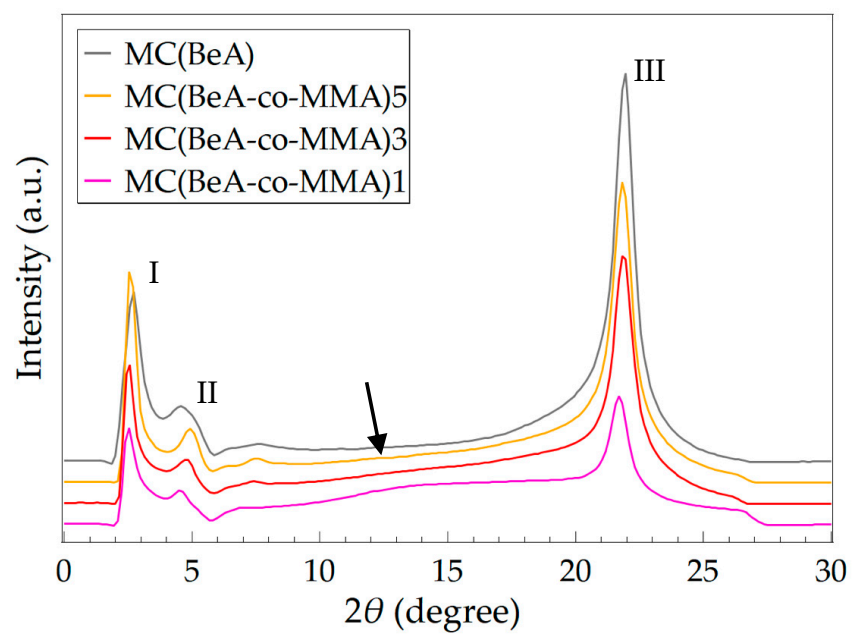

Figure 3. SAXS patterns of $\mathrm{MC}(\mathrm{BeA}-c o-\mathrm{MMA})$ microcapsules and $\mathrm{MC}(\mathrm{BeA})$ measured at room temperature. Three diffraction peaks were shown for both $\mathrm{MC}(\mathrm{BeA})$ and $\mathrm{MC}(\mathrm{BeA}-c o-\mathrm{MMA})$.

Table 3. The locations of diffraction peaks and corresponding interplanar crystal spacing $d$ of $\mathrm{MC}(\mathrm{BeA}-\mathrm{co}-\mathrm{MMA})$ microcapsules and $\mathrm{MC}(\mathrm{BeA})$ calculated from the SAXS results.

\begin{tabular}{|c|c|c|c|c|c|c|}
\hline Sample & 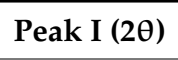 & $d_{1}(\AA)$ & Peak II (2ө) & $d_{2}(\AA)$ & 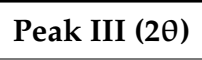 & $d_{3}(\AA)$ \\
\hline $\mathrm{MC}(\mathrm{BeA})$ & 2.72 & 32.45 & 4.58 & 19.28 & 21.95 & 4.05 \\
\hline MC(BeA-co-MMA)5 & 2.55 & 34.62 & 4.93 & 17.91 & 21.82 & 4.07 \\
\hline $\mathrm{MC}(\mathrm{BeA}-c o-\mathrm{MMA}) 3$ & 2.44 & 36.18 & 4.86 & 18.17 & 21.82 & 4.07 \\
\hline MC(BeA-co-MMA) 1 & 2.40 & 36.78 & 4.49 & 19.67 & 21.69 & 4.09 \\
\hline
\end{tabular}

For MC(BeA-co-MMA) microcapsules, it was noted that with the increasing amount of BeA, diffraction Peak I and Peak II at small angles shift to higher angles, indicating a smaller interplanar crystal spacing $d$. This suggested that the peaking of the repeating units of the $n$-behenyl side-chain structure became denser with the decreasing amount of the amorphous component MMA. The PMMA segments with a pendent methyl group $\left(-\mathrm{CH}_{3}\right)$ were hard, which prevented the copolymer chains from rotating freely around the carbon-carbon bond and packing closely in a crystal pattern. Whereas the interplanar crystal spacing (Bragg spacing) of Peak III at wide angles was almost the same and the value was close to that of $\mathrm{MC}(\mathrm{BeA})$, which revealed that the packing type of the $n$-behenyl side-chains was not affected by the monomer ratios. Besides, the addition of BeA caused the increasing intensity of Peak III. The increasing amount of BeA led to an increase in the number of methylene units in the crystals, which promised a better thermal energy storage capacity. For sample MC(BeA-co-MMA)1, there could be seen an amorphous halo around $2 \theta=14^{\circ}$ (indicated by the arrow in Figure 3), which was considered to originate from the correlation between the segregate side-chains and main-chains [14]. All the above results discovered that the increasing amount of BeA had a strong effect on the long spacing structure and the thermal-energy-storage capacity. 


\subsection{Effect of Temperature Changing}

MC(BeA-co-MMA) microcapsules exhibit a complicated transition behavior between the isotropic phase and crystal phase. A temperature variable SAXS of MC(BeA-co-MMA) microcapsules and $\mathrm{MC}(\mathrm{BeA})$ were carried out in the cooling process at a slow rate of $0.5^{\circ} \mathrm{C} \cdot \mathrm{min}^{-1}$ from 70 to $35^{\circ} \mathrm{C}$ shown in Figure 4. In order to give a clear understanding and a direct comparison, the scale of intensity of each diagram is set to the same. The higher intensity and smaller half-peak width indicates a better crystal pattern with fewer disturbances from the main-chain skeleton [17,35]. For MC(BeA-co-MMA)5 and MC(BeA-co-MMA)3, the strong diffraction peaks suggested good crystallization properties, which ensured the high phase change enthalpy and good energy storage capacity. In our previous work, an infrared thermal testing was used to evaluate the thermoregulation property $[18,20]$. Owing to the good crystallization property, MC(BeA-co-MMA) 5 and MC(BeA-co-MMA) 3 effectively prolonged the time to reach the setting temperature, showing the huge capacity to store thermal energy.

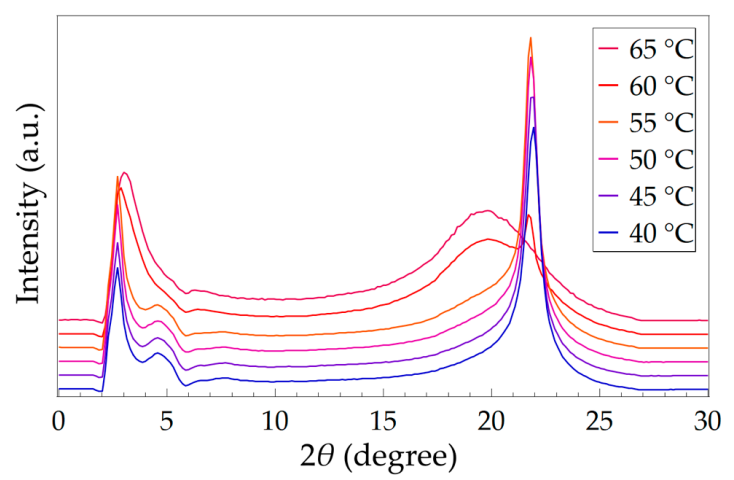

(a) $\mathrm{MC}(\mathrm{BeA})$

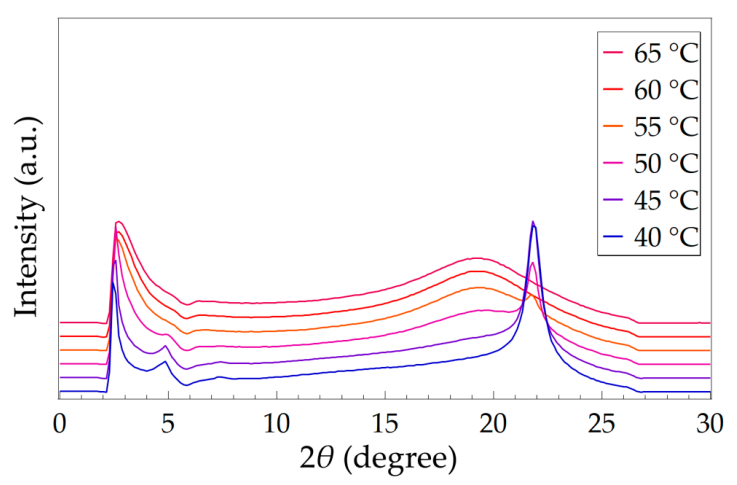

(c) $\mathrm{MC}(\mathrm{BeA}-\mathrm{co}-\mathrm{MMA}) 3$

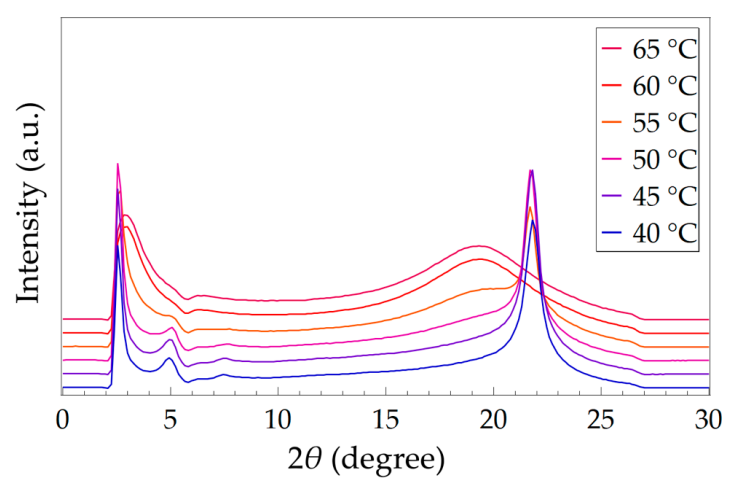

(b) $\mathrm{MC}(\mathrm{BeA}-\mathrm{co}-\mathrm{MMA}) 5$

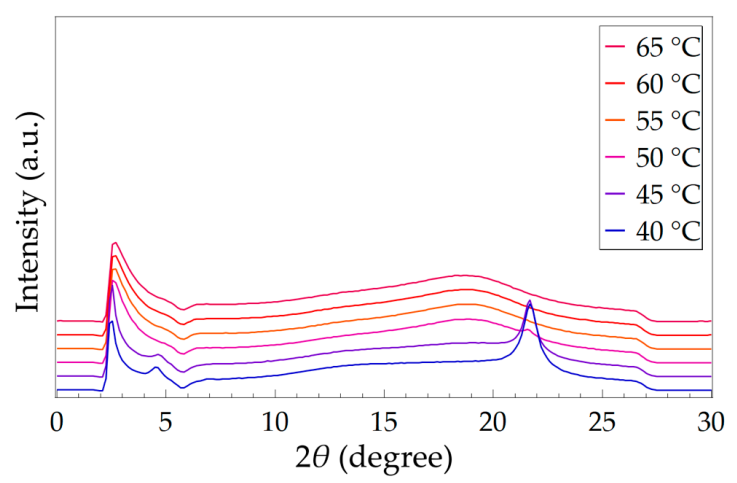

(d) $\mathrm{MC}(\mathrm{BeA}-\mathrm{co}-\mathrm{MMA}) 1$

Figure 4. SAXS patterns of MC(BeA-co-MMA) microcapsules and $\mathrm{MC}(\mathrm{BeA})$ in the cooling process from the isotropic phase to the crystal phase at a cooling rate of $0.5^{\circ} \mathrm{C} \cdot \mathrm{min}^{-1}$. The scale of intensity for each diagram is the same, aiming to give a clear understanding and direct comparison.

MC(BeA-co-MMA) microcapsules showed a broad diffraction peak of the isotropic phase at $2 \theta=19.82^{\circ}$ for $\mathrm{MC}(\mathrm{BeA}), 19.32^{\circ}$ for $\mathrm{MC}(\mathrm{BeA}-\mathrm{co}-\mathrm{MMA}) 5,19.31^{\circ}$ for $\mathrm{MC}(\mathrm{BeA}-\mathrm{co}-\mathrm{MMA}) 3$ and $18.38^{\circ}$ for $\mathrm{MC}(\mathrm{BeA}-\mathrm{Co}-\mathrm{MMA}) 1)$ caused by the disorder accumulation of the $n$-behenyl side-chains. Those strong peaks at the smaller angle of $2 \theta=3.30^{\circ}$ for $\mathrm{MC}(\mathrm{BeA}), 2.84^{\circ}$ for $\mathrm{MC}(\mathrm{BeA}-\mathrm{co}-\mathrm{MMA}) 5,2.72^{\circ}$ for $\mathrm{MC}(\mathrm{BeA}-\mathrm{co}-\mathrm{MMA}) 3$ and $2.70^{\circ}$ for $\left.\mathrm{MC}(\mathrm{BeA}-\mathrm{co}-\mathrm{MMA}) 1\right)$ were also observed. Due to the tough and rigid property of PMMA segments, the interplanar spacing reduced with the decreasing amount of MMA. Therefore, the $n$-behenyl side-chains were found to transit into a crystal state at around $60^{\circ} \mathrm{C}$ for MC(BeA-co-MMA)5, $55^{\circ} \mathrm{C}$ for MC(BeA-co-MMA)3 and $50{ }^{\circ} \mathrm{C}$ for MC(BeA-co-MMA)1. These temperatures were close to the starting point of the corresponding exothermic peak of microcapsules 
according to DSC results. They showed that MC(BeA-co-MMA) microcapsules presented the exothermic peaks in the temperature range of 58 to $41^{\circ} \mathrm{C}$ in the cooling process.

It is noted that within about $5{ }^{\circ} \mathrm{C}$ lower than the side-chain transition point, these peaks at the small angle area near $2 \theta=4.5$ to 5.0 appeared, which were close to the Peak II mentioned above in Figure 3. The peak corresponded to the long spacing order of copolymer chains separated by the side-chains. The copolymer main-chain structure could only transit into the solid phase at a lower temperature. In case of $\mathrm{MC}(\mathrm{BeA}-\mathrm{co}-\mathrm{MMA}) 1$, the broad weak peak around $2 \theta=14^{\circ}$ appeared when the temperature cools down to $45^{\circ} \mathrm{C}$, which was similar to the temperature where the weak shoulder peak appeared on its DSC curve (Figure 2). It indicated that the correlation between the segregate side-chains and main-chains contributes to the phase transition. MMA units worked like defects in the side-chain crystal structure causing irregularity and reducing the flexibility of copolymer as a hard segment. As the amount of MMA decreases, less restriction was caused by the main-chain skeleton, and more methylene units of the $n$-behenyl side-chain could be arranged into crystals freely. This contributed to a higher phase change enthalpy and crystallinity of MC(BeA-co-MMA) microcapsules, so that provided a better energy storage capacity and thermoregulation property.

\subsection{Effect of Temperature Changing Rate}

The regularity of the repeating units strongly affects the crystal transition behavior of $\mathrm{MC}(\mathrm{BeA}-\mathrm{co}-\mathrm{MMA})$ microcapsules. DSC tests of MC(BeA-co-MMA)3 measured at different cooling/ heating rates, including $1^{\circ} \mathrm{C} \cdot \mathrm{min}^{-1}, 3{ }^{\circ} \mathrm{C} \cdot \mathrm{min}^{-1}, 5^{\circ} \mathrm{C} \cdot \mathrm{min}^{-1}$ and $10^{\circ} \mathrm{C} \cdot \mathrm{min}^{-1}$, were used to evaluate the crystal transition behavior. The relative DSC curves are shown in Figure 5, and the detailed data of the phase change temperature and phase transition enthalpy are listed in Table 4 . In the temperature range of 40 to $60^{\circ} \mathrm{C}, \mathrm{MC}(\mathrm{BeA}-c o-\mathrm{MMA}) 3$ displayed a different crystal transition behavior at various temperature changing rates. With the temperature changing rate increasing from $1{ }^{\circ} \mathrm{C}$ to $10^{\circ} \mathrm{C}$, the crystallization temperature tended to decrease; the same trend also for phase change enthalpy. On the cooling curve of MC(BeA-co-MMA)3 at the temperature changing rate of $1{ }^{\circ} \mathrm{C} \cdot \mathrm{min}^{-1}$, a small peak at $55^{\circ} \mathrm{C}$ was detected, along with the corresponding endothermic peak at $60^{\circ} \mathrm{C}$ in the heating process (indicated by the green arrows in Figure 5 (left) and Figure 5 (right), respectively). These peaks were not detected any more under the condition of a faster temperature changing rate. According to the XRD result of MC(BeA-co-MMA)3 shown in Figure 4c, the initial phase transition temperature of the crystalline $n$-behenyl side-chains was the same around $55^{\circ} \mathrm{C}$. The introduction of a tough and rigid PMMA segments reduced the flexibility of the copolymer chains and the mobility of the crystalline side-chains, and made the copolymer harder to form a crystal. On the heating curves of $\mathrm{MC}(\mathrm{BeA}-\mathrm{co}-\mathrm{MMA}) 3$ at a higher temperature changing rate, a small shoulder endothermic peak was observed below the melting point (indicated by the black arrow in Figure 5 (right)). It revealed the existence of a metastable crystal forming below melting point with a faster cooling process. Melting of a remaining metastable crystal form occurred at a temperature near $48^{\circ} \mathrm{C}$, and then melting of the most stable form occurred.

As discussed in the previous section, the comb-like copolymer with a crystalline long $n$-alkane side-chain attached to the main-chain skeleton packed into a layered structure. The side-chains of the comb-like MC(BeA-co-MMA) microcapsules were aligned perpendicularly to the basal plane on the crystal pattern according to XRD results. The crystal structure of the MC(BeA-co-MMA) copolymer microcapsules with a crystalline $n$-behenyl side-chain are shown in Figure 6 . Their crystal transition behavior can be interpreted as follows. At a temperature above the melting point, the copolymer was in the isotropic phase. As the temperature was going down, $n$-behenyl side-chains arranged into a crystal packing form, starting semi-crystalline and reaching a stable crystal pattern. Due to the restriction of the rigid copolymer skeleton, only the terminal parts of the $n$-behenyl side-chain could pack into crystals. The increasing amount of BeA allowed methylene units of the $n$-behenyl side-chain to arrange into crystals more freely, and contributed to a higher phase change enthalpy and crystallinity. 

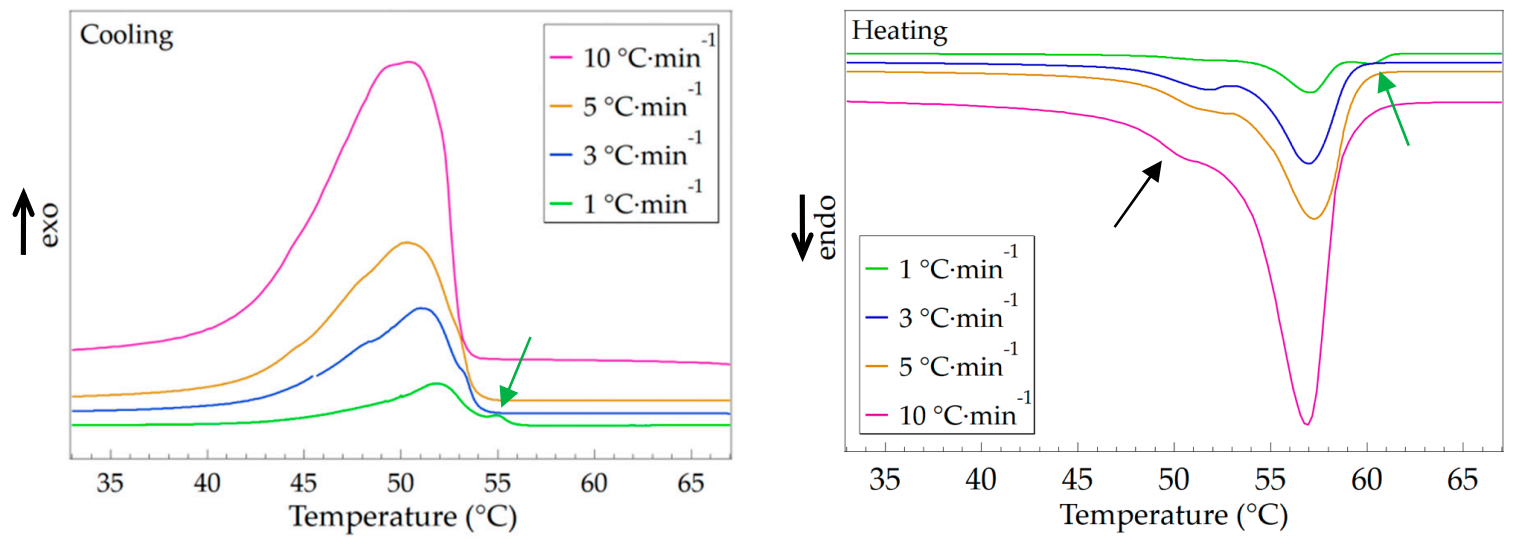

Figure 5. DSC curves of the $\mathrm{MC}(\mathrm{BeA}-\mathrm{co}-\mathrm{MMA}) 3$ microcapsule measured at different temperature changing rates (including $1{ }^{\circ} \mathrm{C} \cdot \mathrm{min}^{-1}, 3^{\circ} \mathrm{C} \cdot \mathrm{min}^{-1}, 5^{\circ} \mathrm{C} \cdot \mathrm{min}^{-1}$ and $10^{\circ} \mathrm{C} \cdot \mathrm{min}^{-1}$ ) in the cooling (left) and heating (right) processes.

Table 4. Thermal properties of the MC(BeA-co-MMA)3 microcapsule measured at different temperature changing rates (including $1{ }^{\circ} \mathrm{C} \cdot \mathrm{min}^{-1}, 3^{\circ} \mathrm{C} \cdot \mathrm{min}^{-1}, 5^{\circ} \mathrm{C} \cdot \mathrm{min}^{-1}$ and $10^{\circ} \mathrm{C} \cdot \mathrm{min}^{-1}$ ).

\begin{tabular}{|c|c|c|c|c|}
\hline \multirow{2}{*}{$\begin{array}{l}\text { Temperature } \\
\text { Changing Rate }\end{array}$} & \multicolumn{2}{|c|}{ Crystallization } & \multicolumn{2}{|c|}{ Melting } \\
\hline & $T_{\mathrm{c}}\left({ }^{\circ} \mathrm{C}\right)$ & $\Delta H_{\mathrm{c}}\left(\mathrm{J} \cdot \mathrm{g}^{-1}\right)$ & $T_{\mathrm{m}}\left({ }^{\circ} \mathrm{C}\right)$ & $\Delta H_{\mathrm{m}}\left(\mathrm{J} \cdot \mathrm{g}^{-1}\right)$ \\
\hline $1^{\circ} \mathrm{C} \cdot \min ^{-1}$ & 51.8 & 112.5 & 57.1 & 114.7 \\
\hline $3^{\circ} \mathrm{C} \cdot \mathrm{min}^{-1}$ & 51.1 & 105.8 & 56.9 & 106.3 \\
\hline $5{ }^{\circ} \mathrm{C} \cdot \min ^{-1}$ & 50.4 & 105.2 & 57.3 & 104.9 \\
\hline $10^{\circ} \mathrm{C} \cdot \mathrm{min}^{-1}$ & 50.3 & 102.1 & 56.9 & 103.1 \\
\hline
\end{tabular}

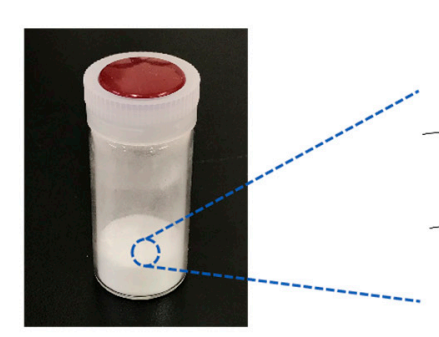

MC(BeA-co-MMA) microcapsules
Release

(crystallization)

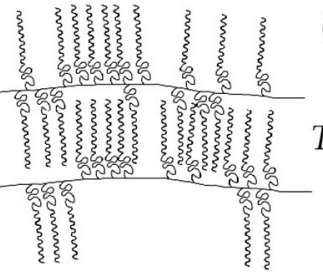

Crystal phase
Store

(melting)

Thermal energy

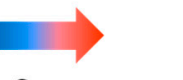
)

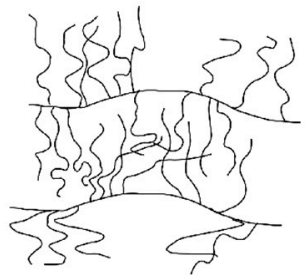

Figure 6. Schematic diagram of the phase transition behavior and crystal structure of MC(BeA-co-MMA) microcapsules with a crystalline $n$-behenyl side-chain.

\subsection{Effect of Synthesis Method}

By adjusting the monomer ratio of the copolymer, microcapsules with varying phase transition behavior and thermal properties can be prepared. Using copolymer microcapsules and sheets with the same ingredients as samples, we investigated the effects of synthesis method on the thermal and crystallization performance of the copolymer materials. Monomer BeA and MMA can form copolymer sheets under UV irradiation through free-radical light-induced polymerization. The DSC curves of ST(BeA-co-MMA) copolymer sheets are shown in Figure 7, and the detailed data are listed in Table 5. 


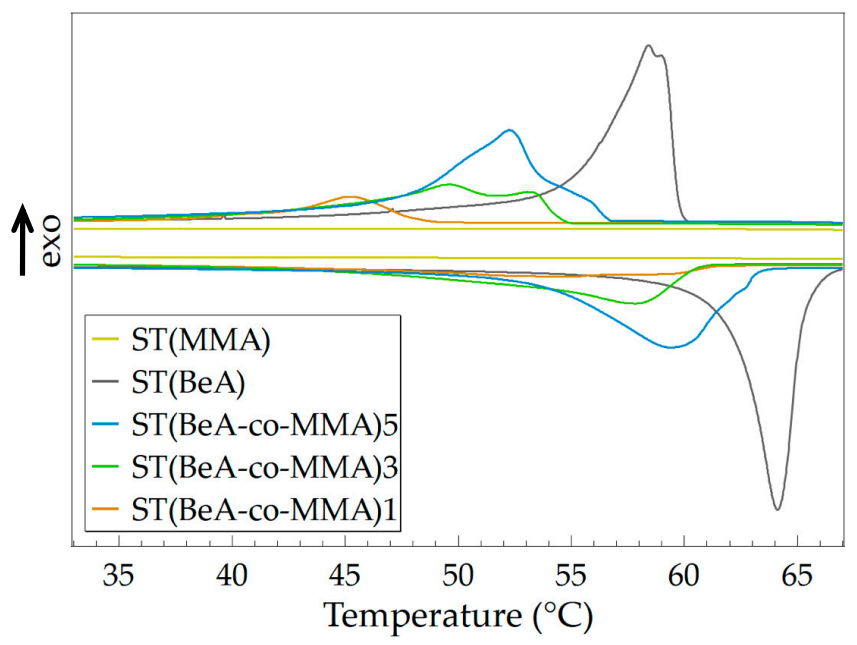

Figure 7. DSC curves of sheet samples for ST(BeA-co-MMA) copolymers with different monomer ratios and homopolymers ST(MMA) and ST(BeA) measured at a temperature changing rate of $5{ }^{\circ} \mathrm{C} \cdot \mathrm{min}^{-1}$.

Table 5. Thermal properties of sheet samples measured at a temperature changing rate of $5{ }^{\circ} \mathrm{C} \cdot \mathrm{min}^{-1}$ for the ST(BeA-co-MMA) copolymer with different monomer ratios and homopolymers ST(MMA) and $\mathrm{ST}(\mathrm{BeA})$.

\begin{tabular}{cccccc}
\hline \multirow{2}{*}{ Sample } & \multicolumn{2}{c}{ Crystallization } & \multicolumn{2}{c}{ Melting } & Degree of \\
\cline { 2 - 5 } & $\boldsymbol{T}_{\mathbf{c}}\left({ }^{\circ} \mathbf{C}\right)$ & $\boldsymbol{\Delta H}_{\mathbf{c}}\left(\mathbf{J} \cdot \mathbf{g}^{-\mathbf{1}}\right)$ & $\boldsymbol{T}_{\mathbf{m}}\left({ }^{\circ} \mathbf{C}\right)$ & $\boldsymbol{\Delta} \boldsymbol{H}_{\mathbf{m}}\left(\mathbf{J} \cdot \mathbf{g}^{-\mathbf{1}}\right)$ & Supercooling $\left({ }^{\circ} \mathbf{C}\right)$ \\
\hline ST(MMA) & - & - & - & - & - \\
ST(BeA) & 58.4 & 115.2 & 64.1 & 115.9 & 5.7 \\
ST(BeA-co-MMA)5 & 52.3 & 91.0 & 59.4 & 91.0 & 7.1 \\
ST(BeA-co-MMA)3 & 49.7 & 61.6 & 57.8 & 61.4 & 8.1 \\
ST(BeA-co-MMA)1 & 45.2 & 23.7 & 55.0 & 25.2 & 9.8 \\
\hline
\end{tabular}

Like microcapsules, the phase change enthalpy and phase transition temperature of the copolymer sheets increased with the increasing amount of phase change component BeA. However, the phase transition behavior of copolymer sheets were different from that of microcapsules. Under the same monomer ratio, the copolymer sheet sample exhibited a lower phase change enthalpy, a lower phase transition temperature and a higher degree of supercooling. The microcapsule samples were synthesized through suspension polymerization, and the yield was around $60 \%$. This indicated a loss of monomers during the polymerization process. Owing to the slight solubility of MMA in water, part of the MMA might dissolve in the water phase. In addition, the emulsion system in suspension polymerization was a little bit unstable, which might also cause a loss. On the other hand, the sheet samples were synthesized through light-induced polymerization. The mixture of monomer BeA, monomer MMA, crosslinker MBAA and initiator BP in a homogeneous phase was injected into a mold. The sheet samples were prepared after $48 \mathrm{~h} \mathrm{UV}$ irradiation. There was no loss because the added resin was included in the sheet one hundred percent. Clearly, the content of MMA in sheet samples were higher. The larger amount of MMA caused a stronger restriction on the mobility of the crystalline $n$-behenyl side-chain, and formed more disturbances in the crystals. This reason explained the above DSC results in that the ST(BeA-co-MMA) copolymer sheets presented weak shoulder peaks and longer melting ranges (Figure 7).

The chemical composition and structure of MC(BeA-co-MMA) and ST(BeA-co-MMA) copolymer materials were evaluated by FTIR spectroscopy, presented in Figure 8. Thermal-energy-storage copolymers synthesized from different methods showed the similar spectrum profile containing a series of characteristic absorption. The copolymers showed strong peaks with double intensive absorption appearing at $2920 \mathrm{~cm}^{-1}$ and $2850 \mathrm{~cm}^{-1}$ owing to the alkyl C-H stretching vibrations of the methylene 
group. The $\mathrm{C}=\mathrm{O}$ stretching vibration of the ester group triggered an absorption band near $1734 \mathrm{~cm}^{-1}$. The peaks near 1260, 1160 and $1130 \mathrm{~cm}^{-1}$, which could be assigned to the $\mathrm{C}-\mathrm{O}$ stretching vibration of the ester group, were typical of acrylic ester. The absorption band at $1444 \mathrm{~cm}^{-1}$, which was attributed to the $\mathrm{C}-\mathrm{H}$ bending vibration of the methyl group on the polymer backbone peak derived from MMA, were detected. Compared to microcapsules, these peaks of sheets at $1444 \mathrm{~cm}^{-1}$ were stronger, and the intensity became weaker with the increasing amount of BeA. It indicated the content of MMA in sheets was higher than that of microcapsules. It matched the DSC results.

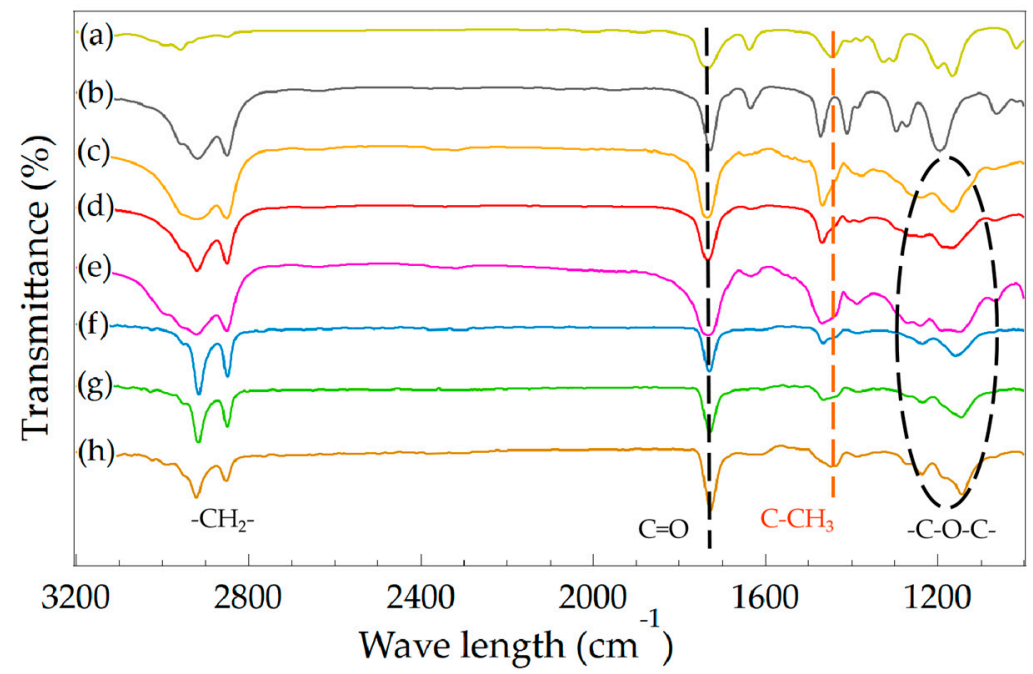

Figure 8. FTIR spectra of microcapsule and sheet samples for BeA-co-MMA copolymer and monomers MMA and BeA. (a) MMA, (b) BeA, (c) MC(BeA-co-MMA)5, (d) MC(BeA-co-MMA)3, (e) MC(BeA-co-MMA)1, (f) ST(BeA-co-MMA)5, (g) ST(BeA-co-MMA)3, and (h) ST(BeA-co-MMA)1.

The crystal structure of ST(BeA-co-MMA) copolymer sheets were further investigated by WAXS analysis. WAXS patterns of these copolymer sheets and homopolymer ST(BeA) are shown in Figure 9. The values of $W_{\mathrm{c}}$ and the location of the strongest peak with the corresponding interplanar crystal spacing $d$ for them are listed in Table 6. Noted that despite synthesis methods were different, the diffraction peaks of the crystal packing of the $n$-behenyl side-chains were around $2 \theta=21.86^{\circ}$, close to that of copolymer microcapsules. When the monomer ratio of BeA to MMA was 1:1, $W_{\mathrm{c}}$ was only $13.39 \%$ (Table 6), and the average $\Delta H$ of $\Delta H_{\mathrm{c}}$ and $\Delta H_{\mathrm{m}}$ was only $24.5 \mathrm{~J} \cdot \mathrm{g}^{-1}$ (Table 5). ST(BeA-co-MMA) 1 could barely provide energy storage capacity because of relatively low $\Delta H$. With the increasing amount of BeA, the crystallinity of copolymer sheets increased and the thermal energy storage capacity improved. The highest $W_{\mathrm{c}}$ and $\Delta H$ reached $38.23 \%$ and $91.0 \mathrm{~J} \cdot \mathrm{g}^{-1}$ under a monomer ratio of BeA to MMA at 5:1. The effect of synthesis method was mainly on the copolymer chemical component, but lightly on the crystal packing of the $n$-behenyl side-chains. ST(BeA-co-MMA) copolymer sheets exhibited complex crystal transition behavior, and the crystalline $n$-behenyl side-chains suffered a stronger restriction from the rigid copolymer skeleton during the crystallization process. 


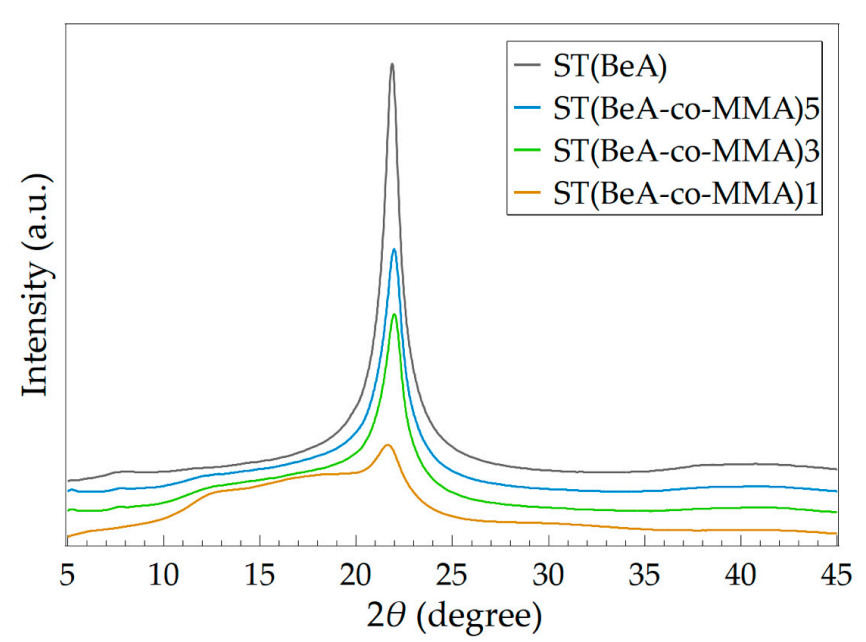

Figure 9. Wide-angle X-ray scatting (WAXS) patterns of ST(BeA-co-MMA) copolymer sheets and homopolymer ST(BeA) measured at room temperature.

Table 6. The location of diffraction peak with its corresponding interplanar crystal spacing $d$, and crystallinity $\left(W_{\mathrm{c}}\right)$ of ST(BeA-co-MMA) copolymer sheets and homopolymer ST(BeA) measured at room temperature.

\begin{tabular}{cccc}
\hline Sample & Peak (20) & $\boldsymbol{d}(\AA)$ & $\boldsymbol{W}_{\mathbf{c}}(\mathbf{\%})$ \\
\hline ST(BeA) & 21.86 & 4.06 & 56.67 \\
ST(BeA-co-MMA)5 & 21.98 & 4.04 & 38.23 \\
ST(BeA-co-MMA)3 & 21.98 & 4.04 & 29.78 \\
ST(BeA-co-MMA)1 & 21.69 & 4.10 & 13.39 \\
\hline
\end{tabular}

\section{Conclusions}

To figure out the correlation between the thermal properties and crystal structure of the thermal-energy-storage BeA-co-MMA copolymer, the effects of monomer ratio, temperature changing and the changing rate, as well as synthesis method, were investigated. Firstly, we synthesized $\mathrm{MC}(\mathrm{BeA}-\mathrm{co}-\mathrm{MMA})$ microcapsules through suspension polymerization. The monomer ratio influenced the crystal transition behavior and thermal properties greatly. The pendent $n$-behenyl group of BeA was highly crystalline, and it acted as a side-chain in the structure of the BeA-co-MMA copolymer. The highly crystalline $n$-behenyl side-chain provided copolymer the thermal-energy-storage capacity. The DSC results proved that when the monomer ratio of BeA and MMA was 3:1, MC(BeA-co-MMA)3 showed the highest average phase change enthalpy $\Delta H\left(105.1 \mathrm{~J} \cdot \mathrm{g}^{-1}\right)$. This indicated that the $n$-behenyl side-chain could form a relatively perfect crystal region ensuring the high energy storage capacity. From the SAXS results, we knew that with the amount of BeA increasing, the interplanar crystal spacing $d$ at small angles became smaller, while the interplanar crystal spacing (Bragg spacing) at wide angles remained almost unchanged. All the above DSC and SAXS results proved that the amount of BeA had a strong effect on the thermal-energy-storage capacity of the copolymer and the long spacing of crystals, but barely on the crystal lamella. We also discussed the effects of temperature changing rate. The results clarified that MMA units worked like defects in the $n$-behenyl side-chain crystal structure of the BeA-co-MMA copolymer. With the decreasing amount of MMA, less restriction was caused by the copolymer skeleton, and more methylene units of the $n$-behenyl side-chain could arrange into crystals freely. That is, a higher fraction of BeA contributed to the higher crystallinity of the $\mathrm{MC}$ (BeA-co-MMA) copolymer, which promised a better energy storage capacity and thermoregulation property. The influence of synthesis method on the crystal transition behavior and thermal properties was investigated by comparing ST(BeA-co-MMA) copolymer sheets prepared through light-induced polymerization with the same ingredients as in MC(BeA-co-MMA). We found that the effect of synthesis 
method was mainly on the copolymer chemical component, but lightly on the crystal packing of the $n$-behenyl side-chains.

Author Contributions: Y.M. and J.G. conceived and designed the experiments; Y.M. performed the experiments; Y.M., J.G., M.Z. and H.I. analyzed the data; Y.M. and J.G. wrote the paper.

Acknowledgments: The financial support of this work from the Asahi Glass Foundation for the Promotion of Science \& Engineering (2016, No.17), the Iketani Science and Technology Foundation (0281059-A), and JSPS KAKENHI (Grant Number 18K05228) are gratefully acknowledged.

Conflicts of Interest: The authors declare no conflicts of interest.

\section{References}

1. Yataganbaba, A.; Ozkahraman, B.; Kurtbas, I. Worldwide trends on encapsulation of phase change materials: A bibliometric analysis (1990-2015). Appl. Energy 2017, 185, 720-731. [CrossRef]

2. Castellon, C.; Martorell, I.; Cabeza, L.F.; Fernandez, A.I.; Manich, A.M. Compatibility of plastic with phase change materials (pcm). Int. J. Energ. Res. 2011, 35, 765-771. [CrossRef]

3. Mondal, S. Phase change materials for smart textiles-an overview. Appl. Therm. Eng. 2008, 28, 1536-1550. [CrossRef]

4. Farid, M.M.; Khudhair, A.M.; Razack, S.A.K.; Al-Hallaj, S. A review on phase change energy storage: Materials and applications. Energy Convers. Manag. 2004, 45, 1597-1615. [CrossRef]

5. Khudhair, A.M.; Farid, M.M. A review on energy conservation in building applications with thermal storage by latent heat using phase change materials. Energy Convers. Manag. 2004, 45, 263-275. [CrossRef]

6. Mahlia, T.M.I.; Saktisahdan, T.J.; Jannifar, A.; Hasan, M.H.; Matseelar, H.S.C. A review of available methods and development on energy storage; technology update. Renew. Sustain. Energy Rev. 2014, 33, 532-545. [CrossRef]

7. Tang, B.T.; Yang, Z.Y.; Zhang, S.F. Poly(polyethylene glycol methyl ether methacrylate) as novel solid-solid phase change material for thermal energy storage. J. Appl. Polym. Sci. 2012, 125, 1377-1381. [CrossRef]

8. Pielichowska, K.; Pielichowski, K. Biodegradable peo/cellulose-based solid-solid phase change materials. Polym. Adv. Technol. 2011, 22, 1633-1641. [CrossRef]

9. Alva, G.; Lin, Y.; Fang, G. Synthesis and characterization of chain-extended and branched polyurethane copolymers as form stable phase change materials for solar thermal conversion storage. Sol. Energy Mater. Sol. Cells 2018, 186, 14-28. [CrossRef]

10. Kenisarin, M.M.; Kenisarina, K.M. Form-stable phase change materials for thermal energy storage. Renew. Sustain. Energy Rev. 2012, 16, 1999-2040. [CrossRef]

11. Karaman, S.; Karaipekli, A.; Sari, A.; Bicer, A. Polyethylene glycol (peg)/diatomite composite as a novel form-stable phase change material for thermal energy storage. Sol. Energy Mater. Sol. Cells 2011, 95, 1647-1653. [CrossRef]

12. Lv, P.; Liu, C.; Rao, Z. Experiment study on the thermal properties of paraffin/kaolin thermal energy storage form-stable phase change materials. Appl. Energy 2016, 182, 475-487. [CrossRef]

13. Zhang, Y.; Wang, L.; Tang, B.; Lu, R.; Zhang, S. Form-stable phase change materials with high phase change enthalpy from the composite of paraffin and cross-linking phase change structure. Appl. Energy 2016, 184, 241-246. [CrossRef]

14. Inomata, K.; Sakamaki, Y.; Nose, T.; Sasaki, S. Solid-state structure of comb-like polymers having n-octadecyl side chains i. Cocrystallization of side chain with n-octadecanoic acid. Polym. J. 1996, 28, 986. [CrossRef]

15. Inomata, K.; Sakamaki, Y.; Nose, T.; Sasaki, S. Solid-state structure of comb-like polymers having n-octadecyl side chains ii. Crystalline-amorphous layered structure. Polym. J. 1996, 28, 992. [CrossRef]

16. Wang, J.; Jian, Y.; Nie, J.; He, Y. Solid photopolymerization and polymer properties of octadecyl vinyl ether. J. Photochem. Photobiol. A 2013, 271, 105-110. [CrossRef]

17. Fallahi, A.; Guldentops, G.; Tao, M.; Granados-Focil, S.; Van Dessel, S. Review on solid-solid phase change materials for thermal energy storage: Molecular structure and thermal properties. Appl. Therm. Eng. 2017, 127, 1427-1441. [CrossRef]

18. Mao, Y.; Gong, J.; Zhu, M.; Ito, H. Excellent thermal stability p(bea-co-mma) microcapsules with high thermal energy storage capacity. Polymer 2018, 150, 267-274. [CrossRef] 
19. Gong, J.; Hosaka, E.; Sakai, K.; Ito, H.; Shibata, Y.; Sato, K.; Nakanishi, D.; Ishihara, S.; Hamada, K. Processing and thermal response of temperature-sensitive-gel(tsg)/polymer composites. Polymers 2018, 10, 486. [CrossRef]

20. Mao, Y.; Miyazaki, T.; Sakai, K.; Gong, J.; Zhu, M.; Ito, H. A 3d printable thermal energy storage crystalline gel using mask-projection stereolithography. Polymers 2018, 10, 1117. [CrossRef]

21. Mao, Y.; Miyazaki, T.; Gong, J.; Zhu, M. Energy storage crystalline gel materials for 3d printing application. Proc. SPIE 2017. [CrossRef]

22. Gong, J.; Azusa, S.; Makino, M.; Kawakami, M.; Furukawa, H. 3d printing of medical and edible gels. J. Jpn. Soc. Abrasive Technol. 2016, 60, 134-137.

23. Gong, J.; Furukawa, H. Smart optical device of varifocal lens developed with high transparent shape memory gels. Expected Mater. Future 2013, 13, 5-8.

24. Gong, J.; Igarashi, S.; Sawamura, K.; Makino, M.; Hasnat Kabir, M.; Furukawa, H. Gel engineering materials meso-decorated with polymorphic crystals. Adv. Mater. Res. 2013, 746, 325-329. [CrossRef]

25. Hu, W. Polymer Physics-a Molecular Approach; Springer: New York, NY, USA, 2013.

26. Ruland, W. X-ray determination of crystallinity and diffuse disorder scattering. Acta Crystallogr. 1961, 14, 1180-1185. [CrossRef]

27. Ryan, A.J.; Bras, W.; Mant, G.R.; Derbyshire, G.E. A direct method to determine the degree of crystallinity and lamellar thickness of polymers: Application to polyethylene. Polymer 1994, 35, 4537-4544. [CrossRef]

28. Yoshio, S.; Kiyoshige, F. Solid-state polymerization of long-chain vinyl compounds. Iv. Effects of chain length on the polymorphic behavior and postpolymerization of $n$-alkyl methacrylates. J. Polym. Sci. 1980, 18, 2437-2494.

29. Shibasaki, Y.; Fukuda, K. Solid-state polymerization of long-chain vinyl compounds. Ii. Effect of molecular arrangement on polymerizability of octadecyl acrylate. J. Polym. Sci. 1979, 17, 2947-2959. [CrossRef]

30. Zhang, Y.; Wang, X.; Wu, D. Design and fabrication of dual-functional microcapsules containing phase change material core and zirconium oxide shell with fluorescent characteristics. Sol. Energy Mater. Sol. Cells 2015, 133, 56-68. [CrossRef]

31. Mukherjee, P.K. Phase transitions among the rotator phases of the normal alkanes: A review. Phys. Rep. 2015, 588, 1-54. [CrossRef]

32. Shibasaki, Y.; Nakahara, H.; Fukuda, K. Solid-state polymerization of long-chain vinyl compounds. I. Effect of molecular arrangement on polymerizability of octadecyl methacrylate. J. Polym. Sci. 1979, 17, 2387-2400. [CrossRef]

33. Sari, A.; Alkan, C.; Bicer, A. Synthesis and thermal properties of polystyrene-graft-peg copolymers as new kinds of solid-solid phase change materials for thermal energy storage. Mater. Chem. Phys. 2012, 133, 87-94. [CrossRef]

34. Şentürk, S.B.; Kahraman, D.; Alkan, C.; Gökçe, İ. Biodegradable peg/cellulose, peg/agarose and peg/chitosan blends as shape stabilized phase change materials for latent heat energy storage. Carbohydr. Polym. 2011, 84, 141-144. [CrossRef]

35. Chen, C.; Liu, W.; Yang, H.; Zhao, Y.; Liu, S. Synthesis of solid-solid phase change material for thermal energy storage by crosslinking of polyethylene glycol with poly(glycidyl methacrylate). Sol. Energ. 2011, 85, 2679-2685. [CrossRef]

(C) 2019 by the authors. Licensee MDPI, Basel, Switzerland. This article is an open access article distributed under the terms and conditions of the Creative Commons Attribution (CC BY) license (http://creativecommons.org/licenses/by/4.0/). 